\title{
A Generalization of the Sum of Divisors Function
}

\author{
Darrell Cox ${ }^{1}$, Sourangshu Ghosh ${ }^{2}$ and Eldar Sultanow ${ }^{3}$ \\ ${ }^{1}$ Department of Mathematics, Grayson County College, United \\ States \\ ${ }^{2}$ Department of Civil Engineering,Indian Institute of Technology \\ Kharagpur, India \\ ${ }^{3}$ Potsdam University, Chair of Business Informatics, Processes and \\ Systems, Potsdam, Germany
}

$\boldsymbol{E}$-mail — Eldar.Sultanow@wi.uni-potsdam.de

Keywords - sum of divisors function, superabundant numbers, colossally abundant numbers, Gronwall's theorem, Riemann hypothesis

\begin{abstract}
A generalization of the sum of divisors function involves a recursive definition. This leads to variants of superabundant numbers, colossally abundant numbers, and Gronwall's theorem (relevant to the Riemann hypothesis).
\end{abstract}

\section{Introduction}

For real or complex $\alpha$ and any integer $n \geq 1$ we define $\sigma_{\alpha}=\sum_{d \mid n} d^{\alpha}$, the sum of the $\alpha$ th powers of the divisors of $n$. The functions $\sigma_{\alpha}$ are called divisor functions. The sum of divisors function is commonly denoted by $\sigma(n)$. The divisor functions are multiplicative and a Dirichlet product of $\sigma(n)$ with the constant function $g(n)=1$ gives another multiplicative function. Similarly, the Dirichet product of this multiplicative function with the constant function gives yet another multiplicative function.

\section{Multiplicative Arithmetical Functions}

An arithmetical function $f$ is called multiplicative if $f$ is not identically zero and if $f(m n)=f(m) f(n)$ whenever $(m, n)=1$. Let $f_{\alpha}(n)=n^{\alpha}$. This function is completely multiplicative ( $m$ and $n$ are not required to be relatively prime). We denote the function $f_{\alpha}$ by $N^{\alpha}$. The divisor functions are multiplicative since $\sigma_{\alpha}=\mu \cdot N^{\alpha}$, the Dirichlet product of two multiplicative functions. The Dirichlet product of arithmetical functions $f$ and $g$ has the form $\sum_{d \mid n} f(d) g\left(\frac{n}{d}\right)$. The 
Möbius function $\mu$ is defined as follows: $\mu(1)=1$. If $n>1$, write $n=p_{1}^{a_{1}} \cdots p_{k}^{a_{k}}$. Then $\mu(n)=(-1)^{k}$ if $a_{1}=a 2=\cdots=a_{k}=1$ or $\mu(n)=0$ otherwise. That the Möbius function is multiplicative is easily seen from its definition.

(1) If $f$ and $g$ are multiplicative, so is their Dirichlet product $f \cdot g$.

See Apostol's [1] Theorem 2.14.

\section{Recursive Definition of Divisor Functions}

Let $r_{n, 1}=\sigma(n), r_{n, 2}=\sum_{d \mid n} r_{d, 1}, r_{n, 3}=\sum_{d \mid n} r_{d, 2}$, etc. Note that these are simplified Dirichlet products so that the functions are multiplicative.

(2) If $n$ is squarefree, $\prod_{p \mid n}(p+1)=r_{n, 1}$. More generally, if $p^{m}$ divides $n$ and $p^{m+1}$ does not divide $n$, then $p^{m}+p^{m-1}+p^{m-2}+\ldots+1$ divides $r_{n, 1}$, that is, $\frac{p^{m+1}-1}{p-1}$ divides $r_{n, 1}$.

(3) If $n$ is squarefree, $\prod_{p \mid n}(p+2)=r_{n, 2}$. More generally, if $p^{m}$ divides $n$ and $p^{m+1}$ does not divide $n$, then $p^{m}+2 p^{m-1}+3 p^{m-2}+\ldots+(m+1)$ divides $r_{n, 2}$, that is, $\frac{\frac{p^{m+2}-1}{p-1}-(m+2)}{p-1}$ divides $r_{n, 2}$.

(4) If $n$ is squarefree, $\prod_{p \mid n}(p+3)=r_{n, 3}$. More generally, if $p^{m}$ divides $n$ and $p^{m+1}$ does not divide $n$, then $p^{m}+3 p^{m-1}+6 p^{m-2}+\ldots+(m+1)(m+2) / 2$ divides $r_{n, 3}$. An expression similar to the above can be derived using the binomial theorem.

\section{Superabundant Numbers}

A natural number is called a superabundant number if $\sigma(n) / n>\sigma(k) / k$ for $1 \leq k \leq n-1$. The first few superabundant numbers are $2,4,6,12,24,36,48$, $60,120,180,240,360,720,840,1260,1680,2520,5040, \ldots$.. Gronwall's theorem (Theorem 323 in Hardy and Wright's [2] book) is

(5) $\lim _{n \rightarrow \infty} \sup \frac{\sigma(n)}{n \log \log n}=e^{\lambda}$

$\lambda$ denotes Euler's constant. A plot of $\frac{\sigma(n)}{n \log \log n}$ for $n=2,4,6,12,24,36,48,60$, $120,180,240,360,720,840,1260,1680,2520,5040,10080,15120,25200,27720$, $55440,110880,166320,277200,332640,554400,665280,720720$, and 1441440 (the superabundant numbers less than 2 million) is 

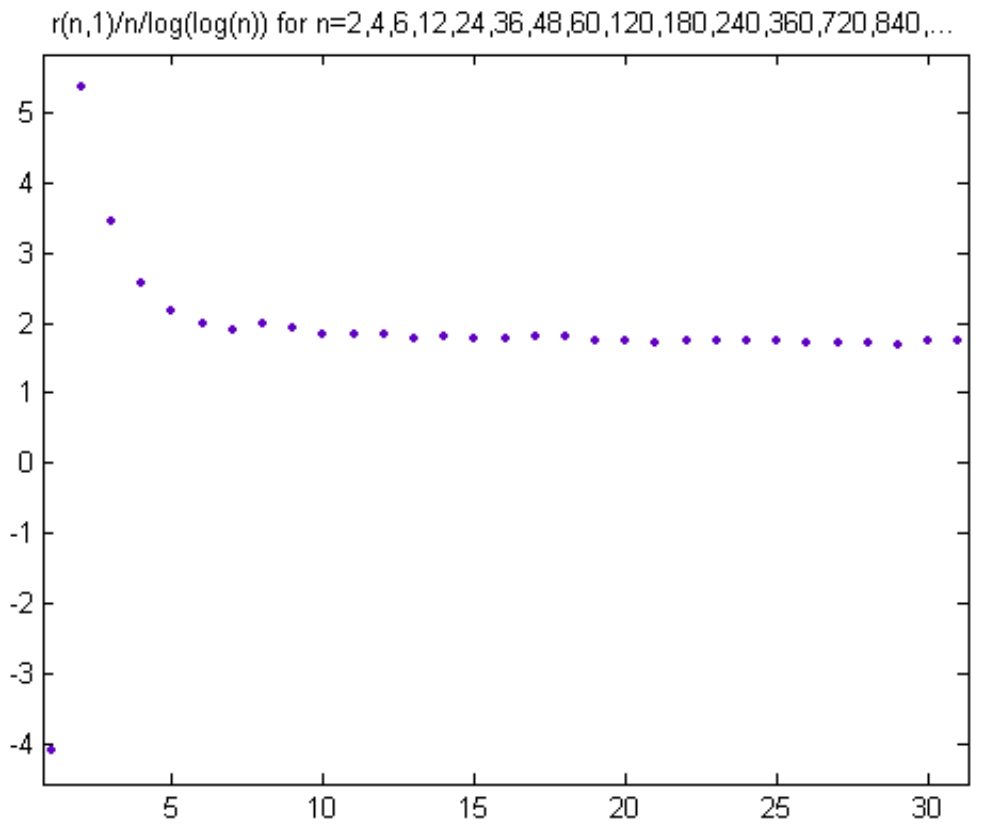

Figure 1: Plot of $\mathrm{r}(\mathrm{n}, 1)$ ratios

$e^{\lambda}$ is approximately equal to 1.7811. A quadratic least-squares fit of $r_{n, 1} / n$ versus $\log \log n$ for these $n$ values is 


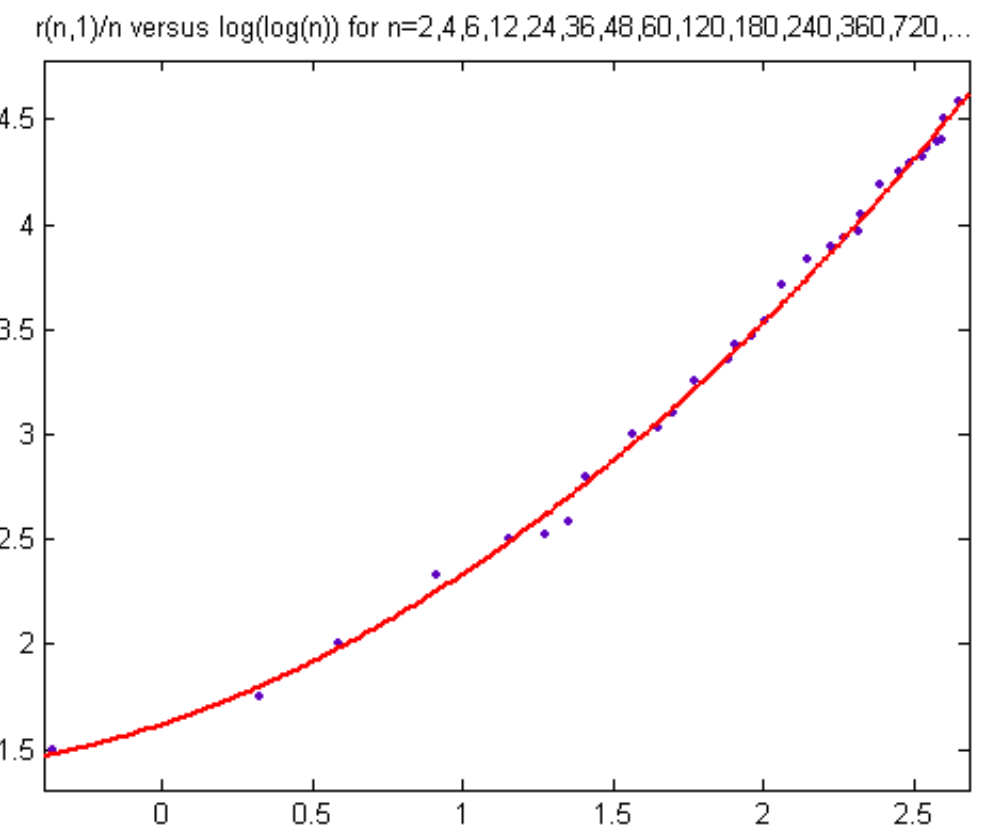

Figure 2: Quadratic least-squares fit

$p_{1}=0.2386$ with a $95 \%$ confidence interval of $(0.21,0.2672), p_{2}=0.4811$ with a $95 \%$ confidence interval of $(0.3966,0.5657), p_{3}=1.62$ with a $95 \%$ confidence interval of $(1.556,1.683), \mathrm{SSE}=0.07629, \mathrm{R}$-squared $=0.9966$, and $\mathrm{RMSE}=0.0522$.

Superabundant numbers consist of a product of all the small primes up to some bound with exponents which are non-increasing as the prime increases. Alaoglu and Erdös' [3] theorems on superabundant numbers are

Theorem 1 If $n=2^{k_{2}} \cdots p^{k_{p}}$, then $k_{2} \geq k_{3} \geq \ldots \geq k_{p}$.

Theorem 2 Let $q<r$, and set $\beta=\left[k_{q} \log q / \log r\right]$. Then $k_{r}$ has one of the three values: $\beta-1, \beta+1, \beta$.

Theorem 3 If $p$ is the largest prime factor of $n$, then $k_{p}=1$, except when $n=4,36$.

In the remainder of the theorems, $p$ always denotes the largest prime factor of $n$.

Theorem 4 If $q$ is either the greatest prime of exponent $k$ or the least prime of exponent $k-1$, and if $q^{1-\theta}>\log p$, then $q^{k}=(p \log p / \log q)\left[1+O\left(\log p / q^{1-\theta} \log q\right)\right]$.

If Riemann's hypothesis is true any $\theta>1 / 2$ can be used. 
Theorem 5 If $k_{q}=k$ and $q<(\log p)^{\alpha}$, where $\alpha$ is a constant, then (i) $\log \frac{q^{k+1}-1}{q^{k+1}-q}>\frac{\log q}{p \log p}\left[1+O\left((\log \log p)^{2} / \log p \log q\right)\right]$, (ii) $\log \frac{q^{k+2}-1}{q^{k+2}-q}<\frac{\log q}{p \log p}[1+$ $\left.O\left((\log \log p)^{2} / \log p \log q\right)\right]$.

Let $K_{q}+1$ be the integral part and $\theta_{q}$ the fractional part of $\log \left[\left(q^{1+\epsilon}-1\right) /\left(q^{\epsilon}-\right.\right.$ $1)] / \log q$, where $\epsilon=\log (1+1 / p) \log p$.

Theorem 6 (i) If $\epsilon_{q}<\theta_{q} \leq 1-\epsilon_{q}$, then $k_{q}=K_{q}$. (ii) If $\theta_{q} \leq \epsilon_{q}$, then $k_{q}=K_{q}$ or $k_{q}=K_{q}-1$. (iii) If $1-\epsilon_{q}<\theta_{q}$, then $k_{q}=K_{q}$ or $K_{q}+1$.

Theorem $7 p \sim \log n$.

Theorem 8 The quotient of two consecutive superabundant numbers tends to 1.

In the proof of Theorem 8 it was shown that the ratio of two consecutive superabundants $n$ and $n^{\prime}$ is less than $1+c(\log \log n)^{2} / \log n$. The order of $(\log p)^{c}$ is used.

Theorem 9 The number of superabundant numbers less than $x$ exceeds $c \log x \log \log x /(\log \log \log x)^{2}$.

Similar superabundant numbers can be defined for $r_{n, 2}, r_{n, 3}, r_{n, 4}$, etc. and usually occur at the same $n$ values. For example, the superabundant numbers for $r_{n, 2}$ satisfy $r_{n, 2} / n>r_{k, 2} / k$ for $1 \leq k \leq n-1$. As for the usual superabundant numbers, these superabundant numbers consist of a product of all the small primes up to some bound with exponents which are non-increasing as the prime increases. Exceptions to Theorem 2 for $r_{n, 2}, r_{n, 3}, r_{n, 4}, r_{n, 5}$, and $r_{n, 6}$ and $n<2000000$ occur at $n=96,480,5760,241920$, and 1209600. In these cases, $k_{r}$ has the value $\beta-2$ for $q=2$ and $r=3$. This conclusion is based on the assumption that the brackets in Theorem 2 denote the floor function. There are also exceptions to Theorem 3 when $q=2$ and $r=3$ (such as when $n=6$, $12,24, \ldots)$. The number of superabundant numbers less than 2000000 for $r_{n, 2}$, $r_{n, 3}, r_{n, 4}, \ldots, r_{n, 12}$ is $38,39,44,43,41,41,42,48,48,49$, and 51 respectively. The number of ordinary superabundant numbers less than 2000000 is 31, so the counts for the new superabundant numbers are consistent with Theorem 9 . All of these theorems should be applicable to the new superabundant numbers. As Alaoglu and Erdös state in the introduction of their article - "In comparing the magnitude of $\sigma(n)$ and $\sigma\left(n^{\prime}\right)$ it is clear from the multiplicative property of $\sigma(n)$ that one need only consider the behavior of those primes which divide the two numbers to different powers. The same is true for $d(n)$ and $\sigma(n) / n$." This appears to be the only property of the sum of divisors function that they use in proving their theorems.

A plot of $\sqrt{\frac{r_{n, 2}}{n \log \log n}}$ for $n=2,4,6,12,24,36,48,60,72,120,180,240$, 360, 720, 1440, 1680, 2160, 2520, 5040, 10080, 15120, 20160, 25200, 30240, 
50400, 55440, 110880, 166320, 221760, 277200, 332640, 554400, 665280, 831600, $1108800,1330560,1441440$, and 1663200 is

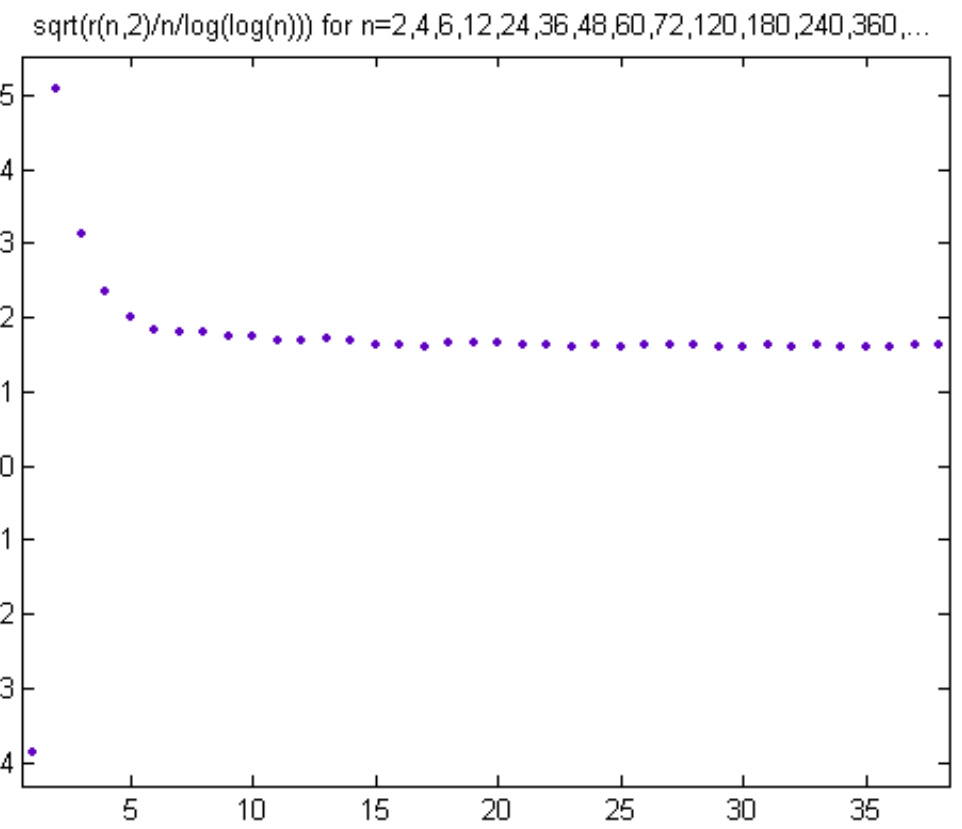

Figure 3: Square root of $\mathrm{r}(\mathrm{n}, 2)$ ratios

In this variant of Gronwall's formula, the supremum limit is unknown but is slightly less than $e^{\lambda}$.

A plot of $\sqrt[3]{\frac{r_{n, 3}}{n \log \log n}}$ for $n=2,4,6,8,12,24,36,48,60,72,120,180,240$, 360, 720, 1440, 2160, 2520, 4320, 5040, 10080, 15120, 20160, 30240, 50400, 55440, 60480, 100800, 110880, 151200, 166320, 221760, 302400, 332640, 554400, $665280,1108800,1330560$, and 1663200 is 

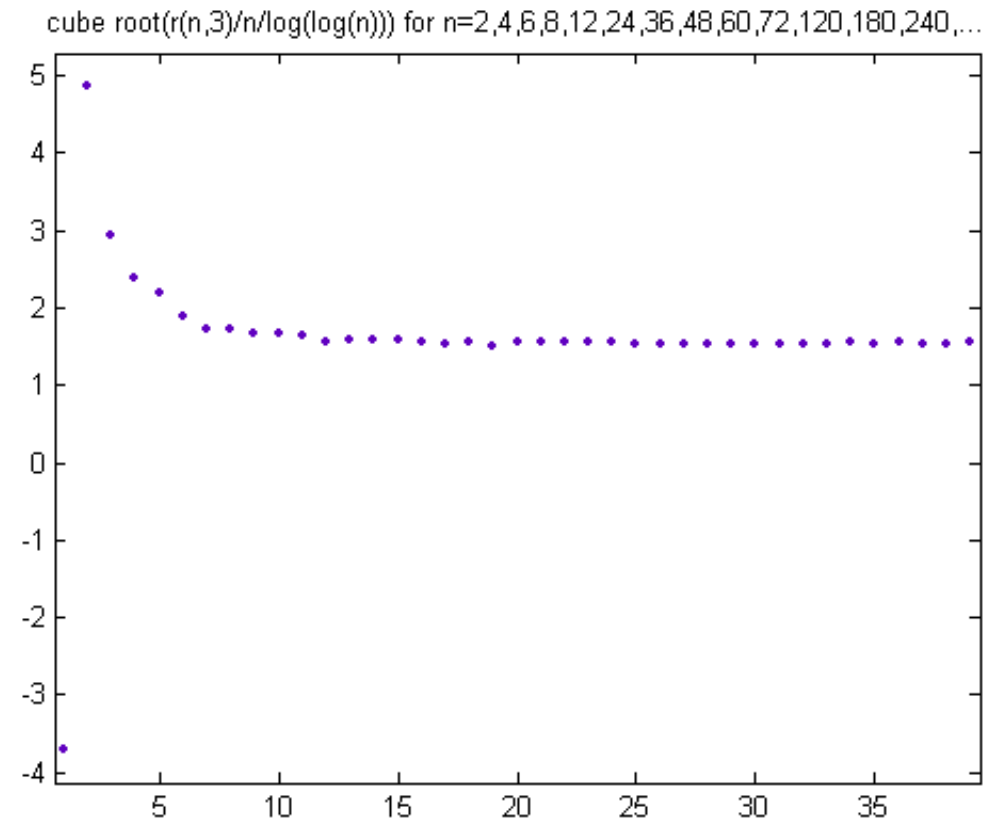

Figure 4: Cube root of $\mathrm{r}(\mathrm{n}, 3)$ ratios

In this variant of Gronwall's formula, the supremum limit is smaller than above.

A plot of $\sqrt[4]{\frac{r_{n, 4}}{n \log \log n}}$ for $n=2,4,6,8,12,24,36,48,72,96,120,144,240,360$, 480, 720, 1440, 2160, 2520, 2880, 4320, 5040, 8640, 10080, 15120, 20160, 30240, 50400, 60480, 100800, 110880, 120960, 151200, 181440, 221760, 302400, 332640, $55440,604800,665280,1108800,1330560,1663200$, and 1995840 is 

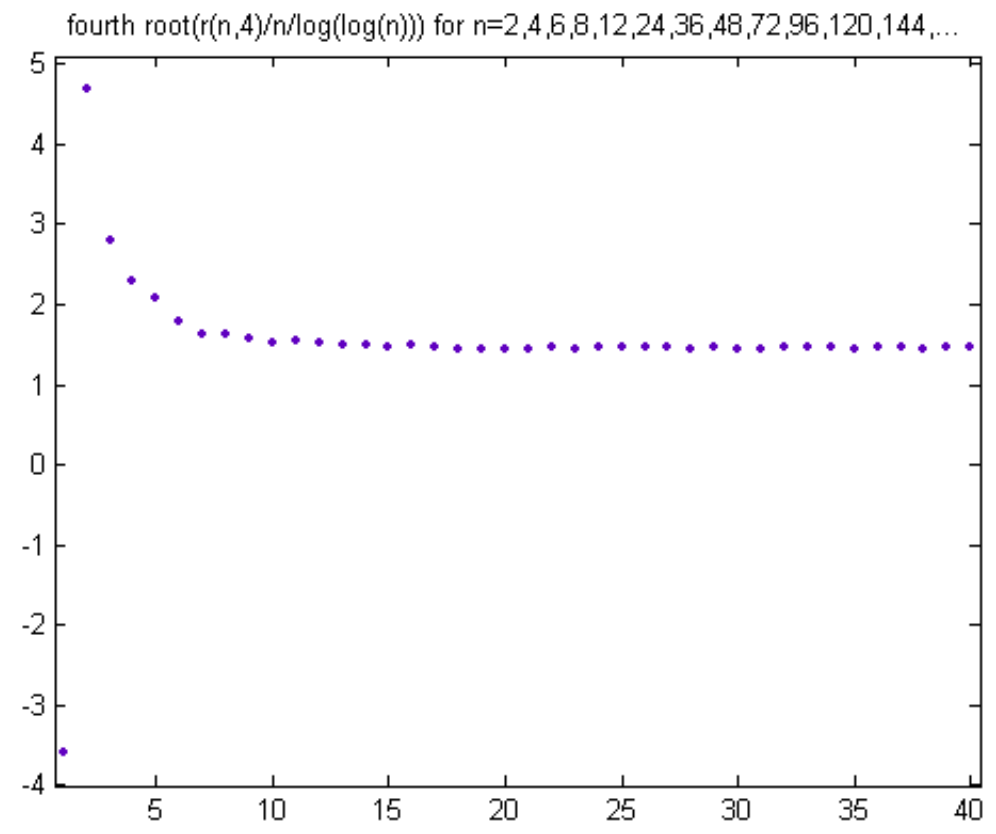

Figure 5: Fourth root of $\mathrm{r}(\mathrm{n}, 4)$ ratios

In this variant of Gronwall's formula, the supremum limit is still smaller.

A quadratic least-squares fit of the smallest positive $\sqrt[i]{\frac{r_{n, i}}{n \log \log n}}$ values for $n<$ 2000000 and $i=1,2,3, \ldots, 12$ is 


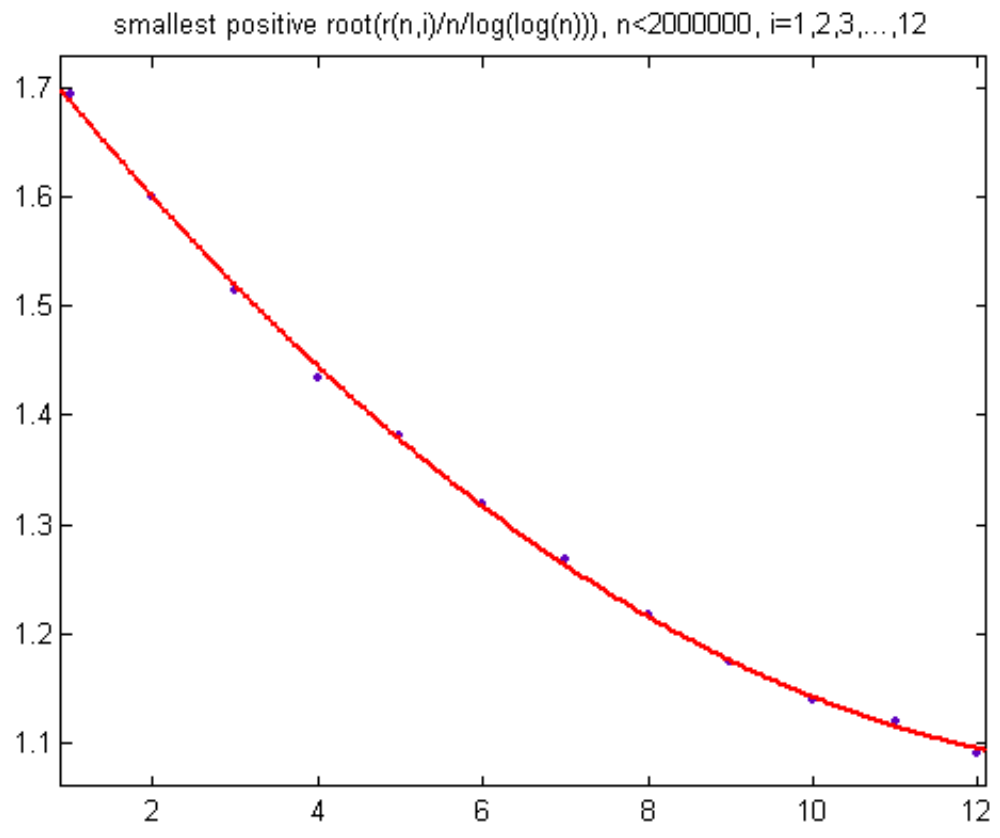

Figure 6: Quadratic least-squares fit

$p_{1}=0.003381$ with a $95 \%$ confidence interval of $(0.003023,0.003739), p_{2}=$ -0.09788 with a $95 \%$ confidence of $(-0.1027,-0.0931), p_{3}=1.782$ with a $95 \%$ confidence interval of $(1.769,1.796), \mathrm{SSE}=0.0003007$, R-squared $=0.9993$, and $\mathrm{RMSE}=0.00578$. The first value is for $\frac{r_{n, 1}}{n \log \log n}$. The values are 1.6943, 1.5998, $1.5135,1.4339,1.3809,1.3190,1.2683,1.2181,1.1736,1.1395,1.1199$, and 1.0902 respectively. For small sample sizes, quadratic curves give good approximations of some logarithmic functions.

A cubic least-squares fit of the smallest positive $\sqrt[i]{\frac{r_{n, i}}{n \log \log n}}$ values for $n<$ 2000000 and $i=1,2,3, \ldots, 16$ is 


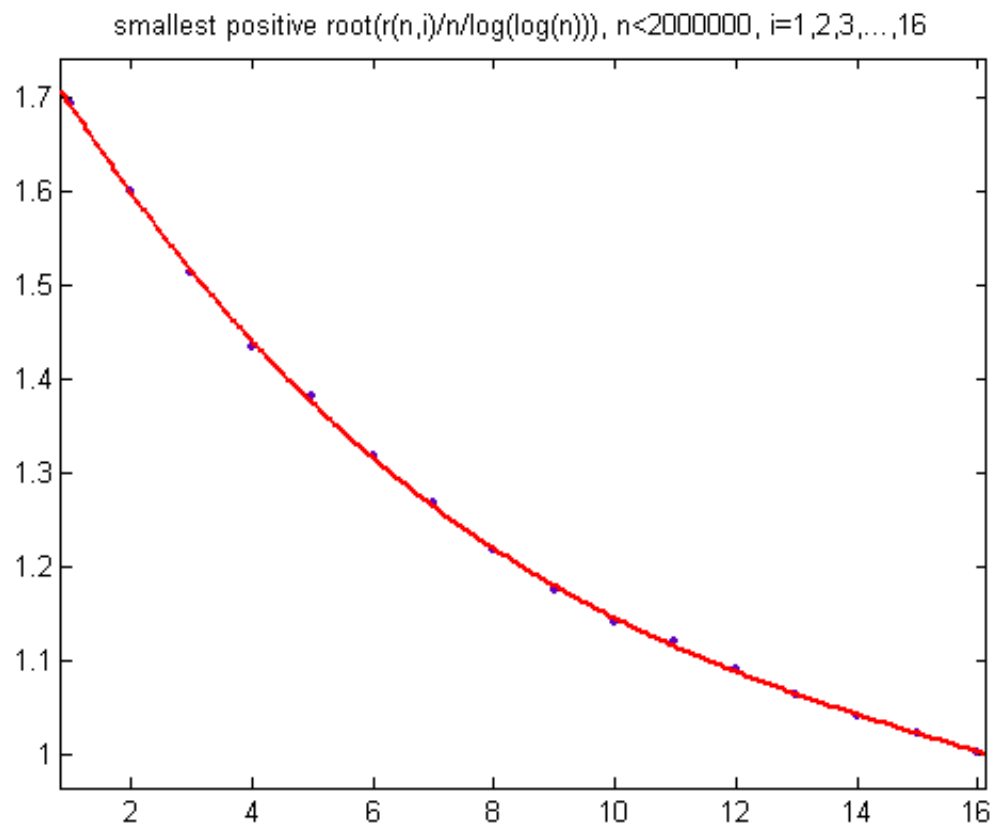

Figure 7: Cubic least-squares fit

$\mathrm{RMSE}=0.0002309, \mathrm{R}$-squared $=0.9997$, and $\mathrm{RMSE}=0.004386$. The values are $1.6943,1.5998,1.5135,1.4339,1.3809,1.3190,1.2683,1.2181,1.1736,1.1395$, $1.1199,1.0902,1.0623,1.0392,1.0278$, and 1.0018 respectively. Eventually, higher order curves will be required to approximate the values (which are expected to approach zero).

\section{Euler's Phi Function and the Sum of Divisors Function}

Hardy and Wright's Theorem 328 is

(6) $\lim _{n \rightarrow \infty} \inf \frac{\phi(n) \log \log n}{n}=e^{-\lambda}$

$\phi(n)$ denotes Euler's phi function. Their Theorem 329 is

(7) $A<\frac{\sigma(n) \phi(n)}{n^{2}}<1$ for a positive constant A.

This relationship between $\phi(n)$ and $\sigma(n)$ is used to prove Gronwall's theorem.

Superabundant numbers can also be defined for the largest $\frac{r_{n, 2} \phi(n)}{n^{2}}$ values. A plot of $\frac{r_{n, 2} \phi(n)}{n^{2} \log \log n}$ for $n=3,4,8,16,24,32,48,72,144,288,432,576,720,864$, 
1440, 2160, 2880, 3600, 4320, 7200, 8640, 10800, 14400, 15120, 20160, 21600, 30240, 43200, 60480, 75600, 100800, 120960, 151200, 302400, 604800, 907200, 1663200 , and 1814400 is

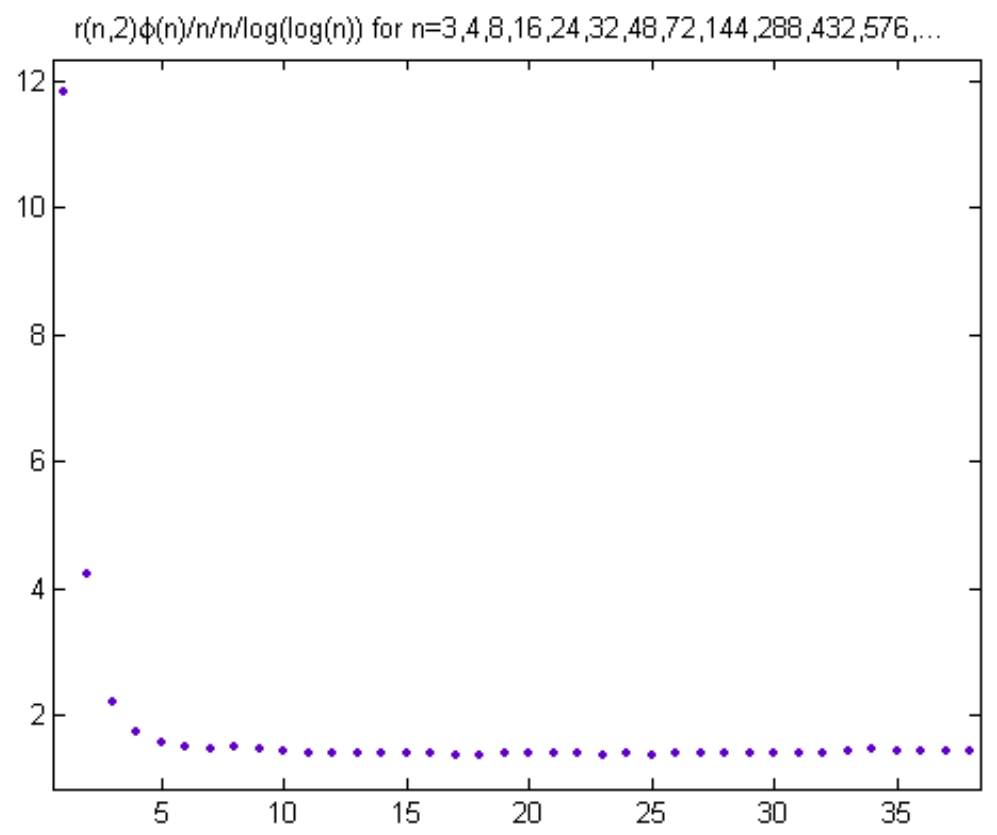

Figure 8: Plot of $\mathrm{r}(\mathrm{n}, 2)$ ratios

In this variant of Gronwall's formula, the supremum limit is unknown. A quadratic least-squares fit of $\frac{r_{n, 2} \phi(n)}{n^{2}}$ versus $\log \log n$ for these $n$ values is 


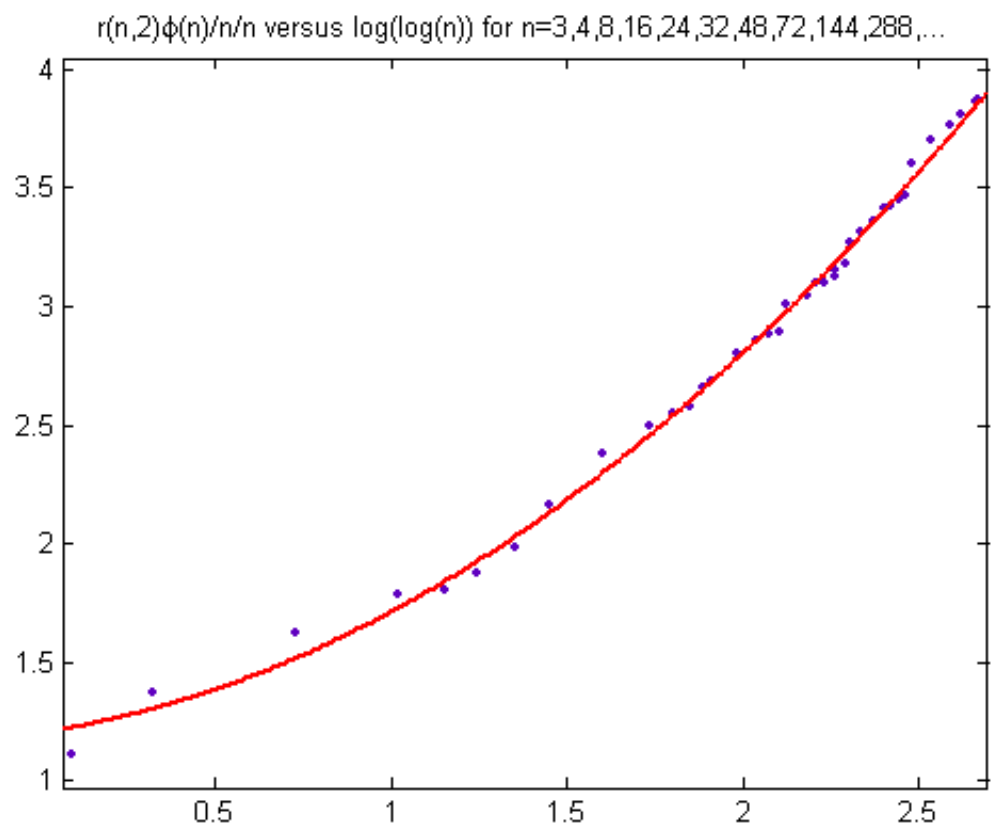

Figure 9: Quadratic least-squares fit of $r_{n, 2}$ ratios

$p_{1}=0.2912$ with a $95 \%$ confidence interval of $(0.2573,0.325), p_{2}=0.2204$ with a $95 \%$ confidence interval of $(0.1131,0.3276), p_{3}=1.201$ with a $95 \%$ confidence interval of $(1.121,1.281), \mathrm{SSE}=0.08065$, R-squared $=0.9958$, and $\mathrm{RMSE}=0.048$. Note the resemblance to the curve in Figure 2.

Superabundant numbers can also be defined for the largest $\frac{r_{n, 3} \phi(n) \phi(n)}{n^{3}}$ values. A plot of $\sqrt{\frac{r_{n, 3} \phi(n) \phi(n)}{n^{3} \log \log n}}$ for $n=5,7,8,16,32,64,128,256,288,432,576$, 864, 1728, 3456, 5184, 6912, 10368, 20736, 31104, 41472, 43200, 86400, 129600, $172800,259200,518400,777600,1036800,1296000$, and 1555200 is 


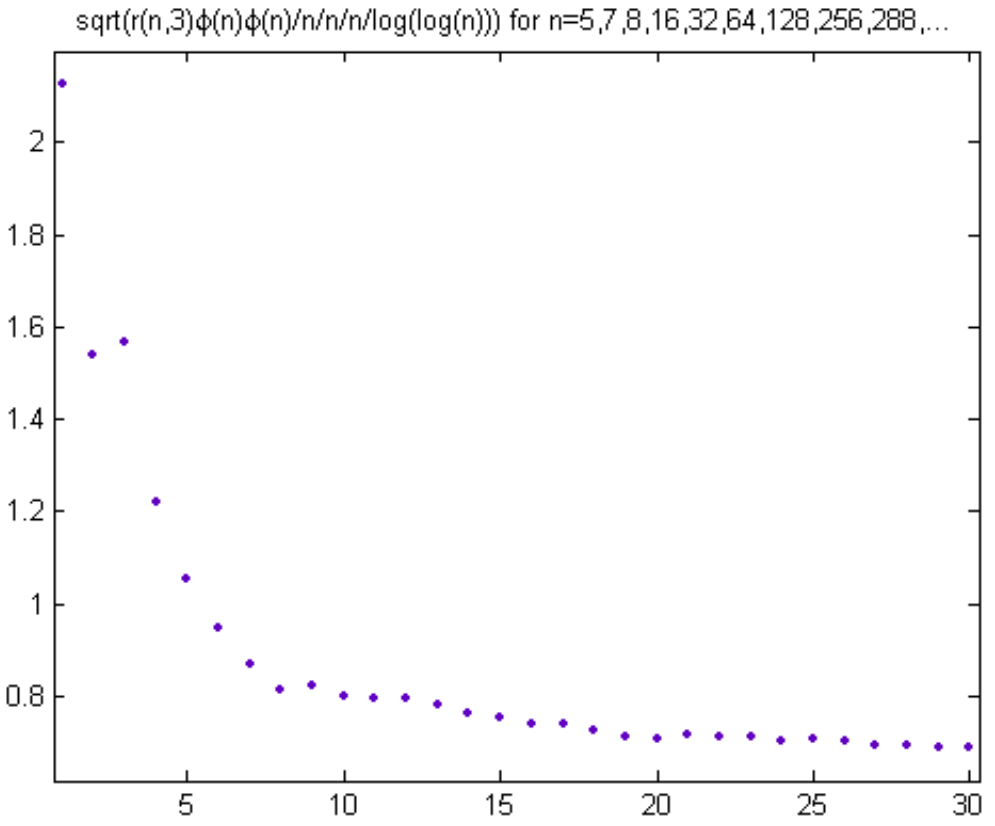

Figure 10: Square root of $\mathrm{r}(\mathrm{n}, 3)$ ratios

Superabundant numbers can also be defined for the largest $\frac{r_{n, 4} \phi(n) \phi(n) \phi(n)}{n^{4}}$ values. A plot of $\sqrt[3]{\frac{r_{n, 4} \phi(n) \phi(n) \phi(n)}{n^{4} \log \log n}}$ for $n=9,11,13,16,32,64,128,256,512,1024$, 1728, 3456, 5184, 6912, 10368, 20736, 31104, 41472, 62208, 124416, 248832, $373248,497664,518400,777600,1036800$, and 1555200 is 


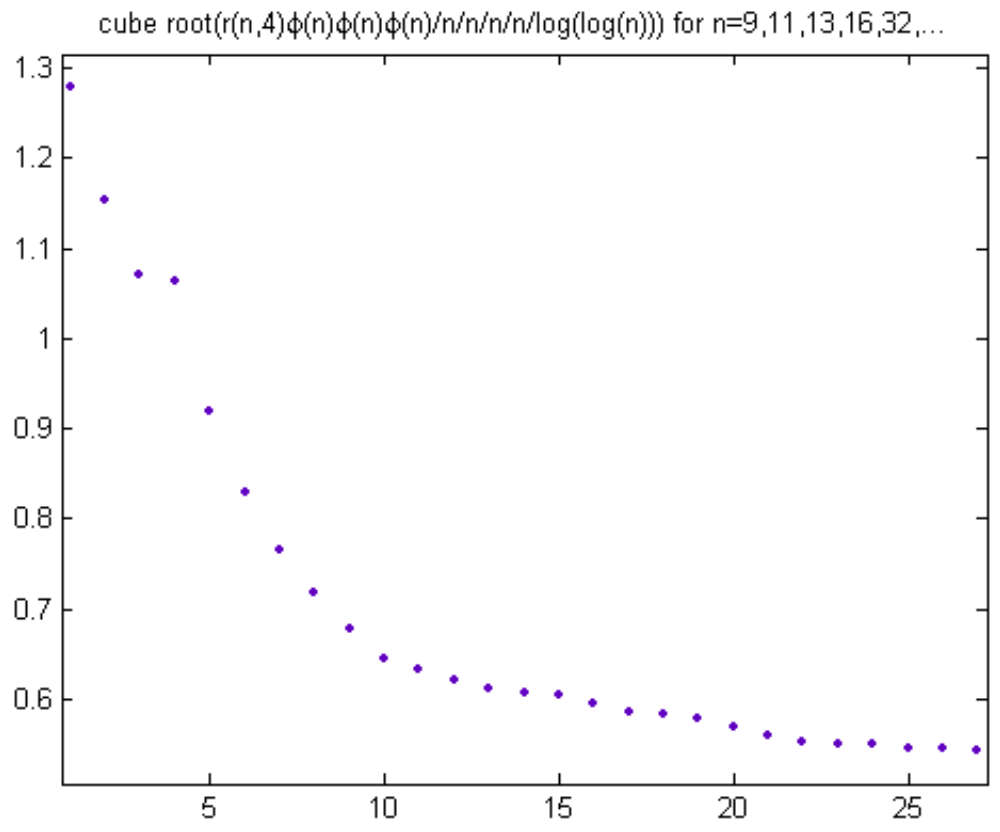

Figure 11: Cube root of $\mathrm{r}(\mathrm{n}, 4)$ ratios

Other superabundant numbers are similarly defined for $r_{n, 5}, r_{n, 6}, r_{n, 7}$, etc. The smallest of the above values for $r_{n, 2}, r_{n, 3}, r_{n, 4}, r_{n, 5}, r_{n, 6}, r_{n, 7}$, and $r_{n, 8}$ are $1.3734,0.6888,0.5427,0.4862,0.4561,0.4350$, and 0.4187 respectively. A plot of the normalized reciprocals of these minima (decremented by 1/1.3734) and $\log (i)$ for $i=1,2,3,4,5,6$, and 7 is 


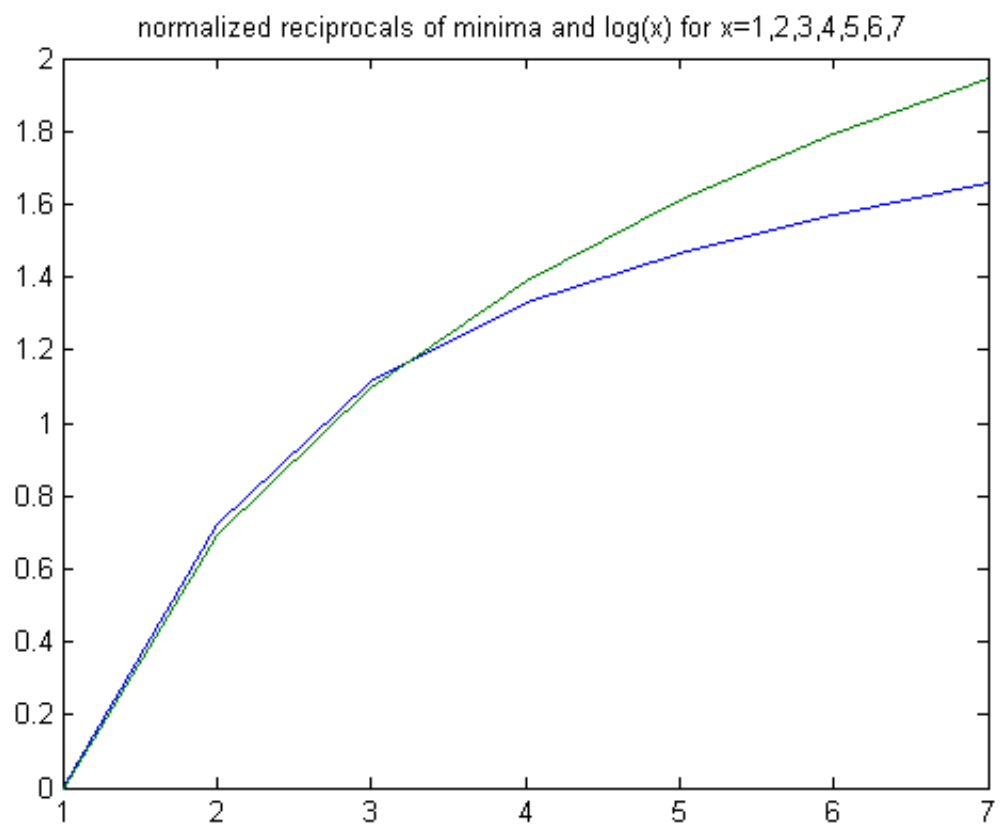

Figure 12: Normalized reciprocals of minimum and $\log (x)$

The logarithm increases more rapidly.

\section{Colossally Abundant Numbers}

In the above, the $n$ values start with a few odd values followed by powers of 2 . Afterwards, the $n$ values are the product of powers of 2 and 3 , the product of powers of 2,3 , and 5 , the product of powers of $2,3,5$, and 7 , etc. For $r_{n, 2}$, the odd numbers and powers of 2 are $3,4,8$, and 16 . For $r_{n, 3}$, the odd numbers and powers of 2 are $5,7,8,16,32,64,128,256$. For $r_{n, 4}$, the odd numbers and powers of 2 are $9,11,13,16,32,64,128,256,512$, and 1024. For $r_{n, 5}$, the odd numbers and powers of 2 are 13, 17, 19, 23, 25, 27, 32, 64, 128, 256, $512,1024,2048,4096$, and 8192. For $r_{n, 6}$, the odd numbers and powers of 2 are $19,23,29,31,37,49,64,128,256,512,1024,2048,4096,8192,16384$, and 32768 . For $r_{n, 7}$, the odd numbers and powers of 2 are 29, 31, 37, 41, 43, 47, 49, $81,125,128,243,256,512,1024,2048,4096,8192,16384,32768,65536$, and 131072. Note that a power of 3 (243) is interspersed between 128 and 256. For $r_{n, 8}$, the odd numbers and powers of 2 are $31,37,41,43,47,53,59,61,67$, $121,125,243,256,512,1024,2048,4096,8192,16384,32768,65536,131072$, 262144,524288 , and 1048576 . For $r_{n, 9}$, the odd numbers and powers of 2 (up to 2 million) are 41, 43, 47, 53, 59, 61, 67, 71, 73, 79, 83, 121, 169, 243, 289, 343, $512,729,1024,2048,4096,8192,16384,32768,65536,131072,262144,524288$, $1048576, \ldots$. Note that a power of 3 (729) is interspersed between 512 and 1024 . 
For $r_{n, 10}$, the odd numbers and powers of 2 (up to 2 million) are $53,59,61,67$, $71,73,79,83,89,97,101,169,289,343,625,729,1024,2048,4096,8192,16384$, $32768,65536,131072,262144,524288,1048576, \ldots$. For $r_{n, 11}$, the odd numbers and powers of 2 (up to 2 million) are $59,61,67,71,73,79,83,89,97,101$, 103, 107, 109, 113, 127, 169, 289, 361, 529, 625, 1331, 2048, 4096, 8192, 16384, $32768,65536,131072,262144,524288,1048576, \ldots$. For $r_{n, 12}$, the odd numbers and powers of 2 (up to 2 million) are $71,73,79,83,89,97,101,103,107,109$, $113,127,131,137,139,149,289,361,529,841,1331,2187,2401,3125,4096$, $6561,8192,16384,32768,65536,131072,262144,524288,1048576, \ldots$. Note that a power of 3 (6561) is interspersed between 4096 and 8192 . For $r_{n, 13}$, the odd numbers and powers of 2 (up to 2 million) are 83, 89, 97, 101, 103, 107, 109, $113,127,131,137,139,149,151,157,163,167,289,361,529,841,1331,2197$, 2401, 3125, 6561, 8192, 15625, 16384, 32768, 65536, 131072, 262144, 524288, $1048576, \ldots$. Note that a power of 5 (15625) is interspersed between 8192 and 16384. For $r_{n, 14}$, the odd numbers and powers of 2 (up to 2 million) are 97, 101, 103, 107, 109, 113, 127, 131, 137, 139, 149, 151, 157, 163, 167, 173, 179, 181, 191, 193, 197, 361, 529, 841, 961, 1331, 2197, 2401, 6561, 14641, 15625, 16384, $32768,65536,131072,262144,524288,1048576, \ldots$. For $r_{n, 15}$, the odd numbers and powers of 2 (up to 2 million) are 113, 127, 131, 137, 139, 149, 151, 157, $163,167,173,179,181,191,193,197,199,221$. 223, 227, 529, 841, 961, 1331, 2197, 4913, 14641, 15625, 19683, 32768, 59049, 65536, 131072, 262144, 524288, $1048576, \ldots$. For $r_{n, 16}$, the odd numbers and powers of 2 (up to 2 million) are $127,131,137,139,149,151,157,163,167,173,179,181,191,193,197,199$, 211, 223, 227, 229, 233, 239, 241, 251, 257, 529, 841, 961, 1369, 1681, 2197, 4913, 6859, 14641, 15625, 16807, 59049, 131072, 262144, 524288, 1048576,... The odd numbers appear to be prime powers. All the $n$ values appear to satisfy Theorem 2 (with the caveat of the $\beta-2$ case). Other than the odd values, the $n$ values behave like colossally abundant numbers. Colossally abundant numbers are a subset of the superabundant numbers.

A spectrum similar to the Riemann spectrum can be computed for the odd numbers up to the first even number by using the function $F_{\leq C}(t)=-\sum_{p^{n} \leq C} \frac{\log (p)}{p^{n / 2}} \cos \left(t \log \left(p^{n}\right)\right)$. A plot of this function for $r_{n, 9}$ and $t \leq 300$ is 


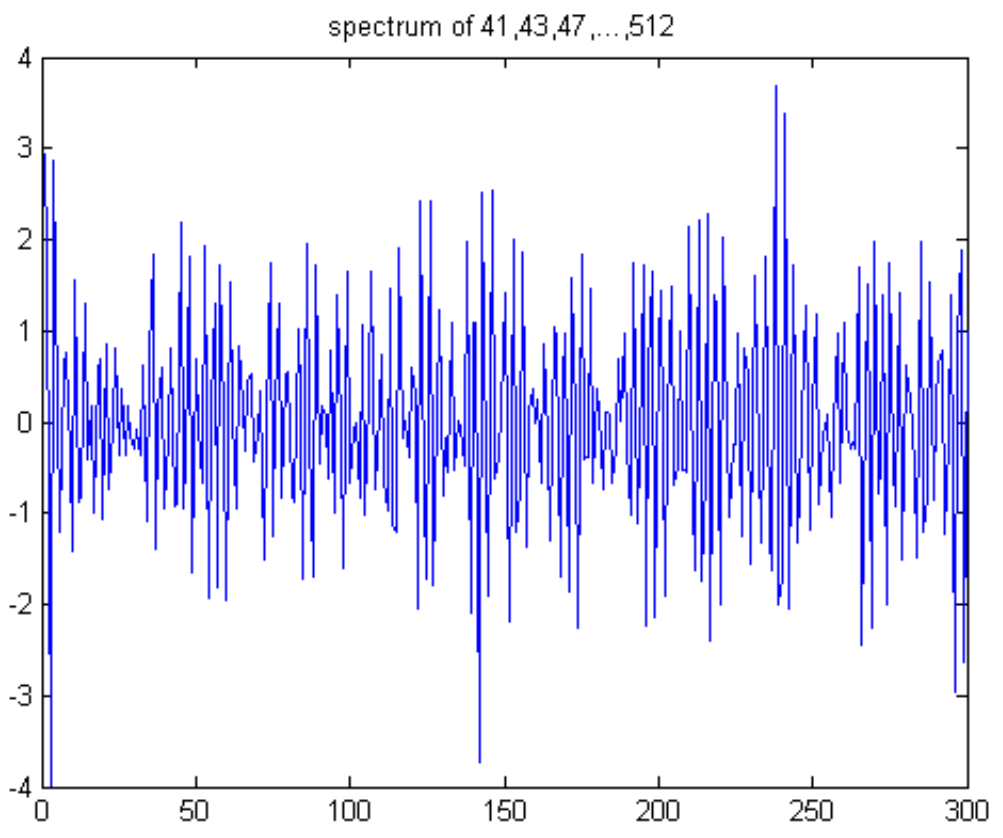

Figure 13: spectrum

The inverse function for the Riemann spectrum is $H_{\leq C}(s)=1+\sum_{i \leq C} \cos \left(\log (s) \theta_{i}\right)$. The non-trivial zeta function zeros are denoted by $\theta_{i}$. In the application of this function here, no maxima of the spectrum are identified since they don't correspond to the prime power locations very well. (Even for the usual prime power sequence, the maxima don't correspond to the prime power locations very well for small cutoffs. See Chapter 30 of Mazur and Stein's [4] book for some good graphs of the Riemann spectrum.) A plot of the inverse function is 


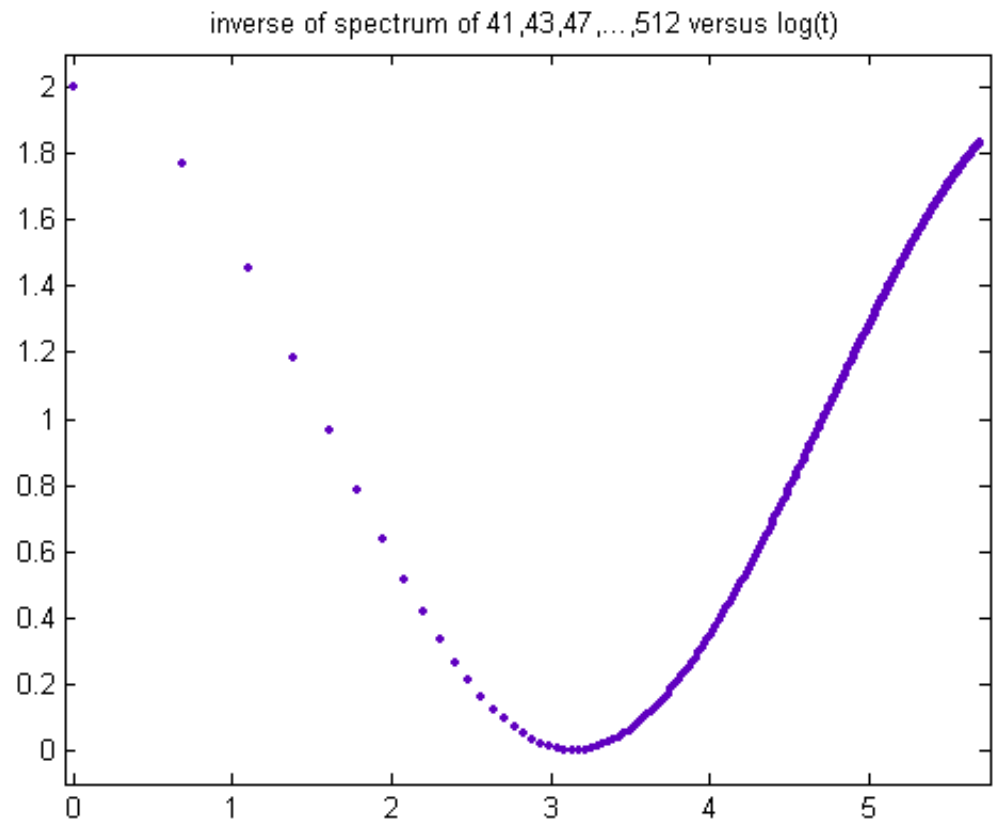

Figure 14: inverse of spectrum

The curve is sinusoidal (at least when larger $t$ values are used). A plot of the inverse function for $r_{n, 10}$ and $t \leq 300$ is 


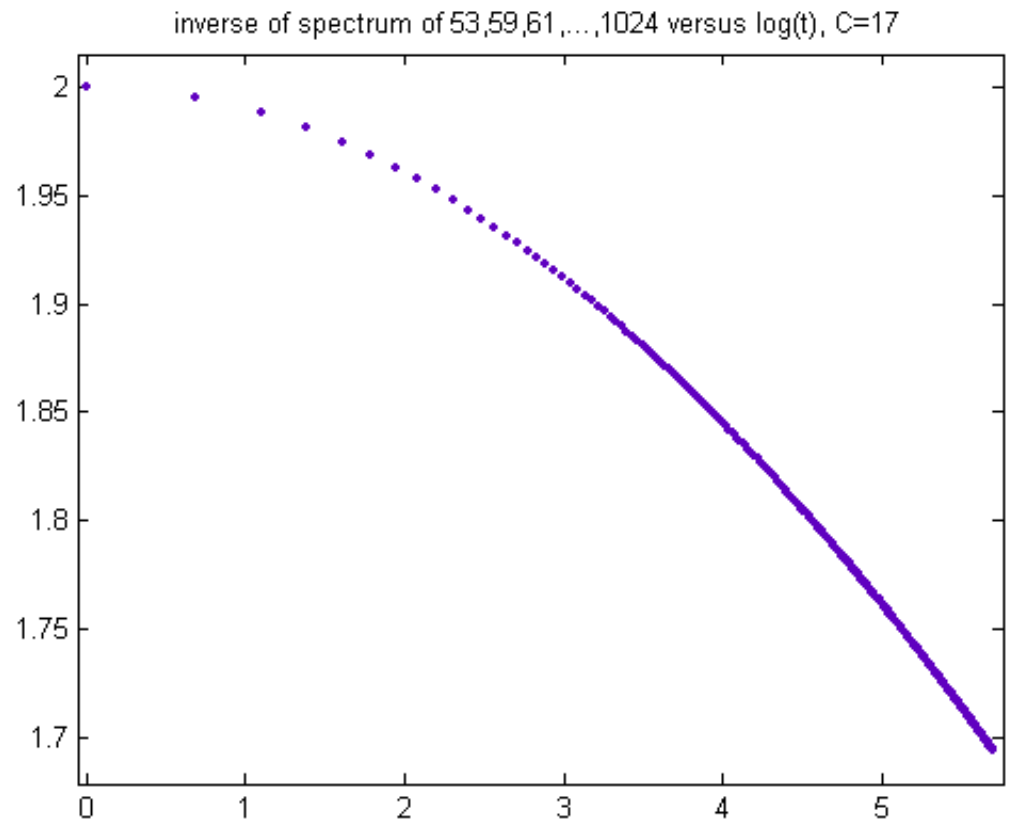

Figure 15: inverse of spectrum

Due to the shape of the curve, the values do not oscillate for larger $t$. A plot of the inverse function for $r_{n, 11}$ and $t \leq 300$ is 


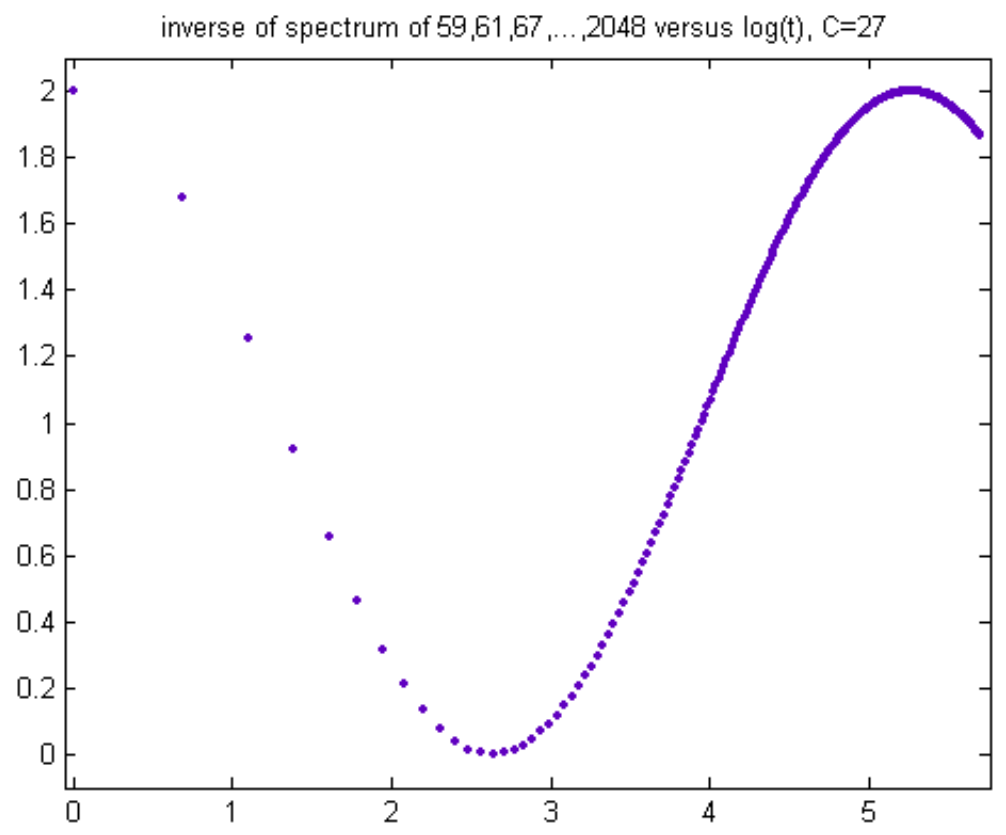

Figure 16: inverse of spectrum

A plot of the inverse function for $r_{n, 12}$ and $t \leq 300$ is 


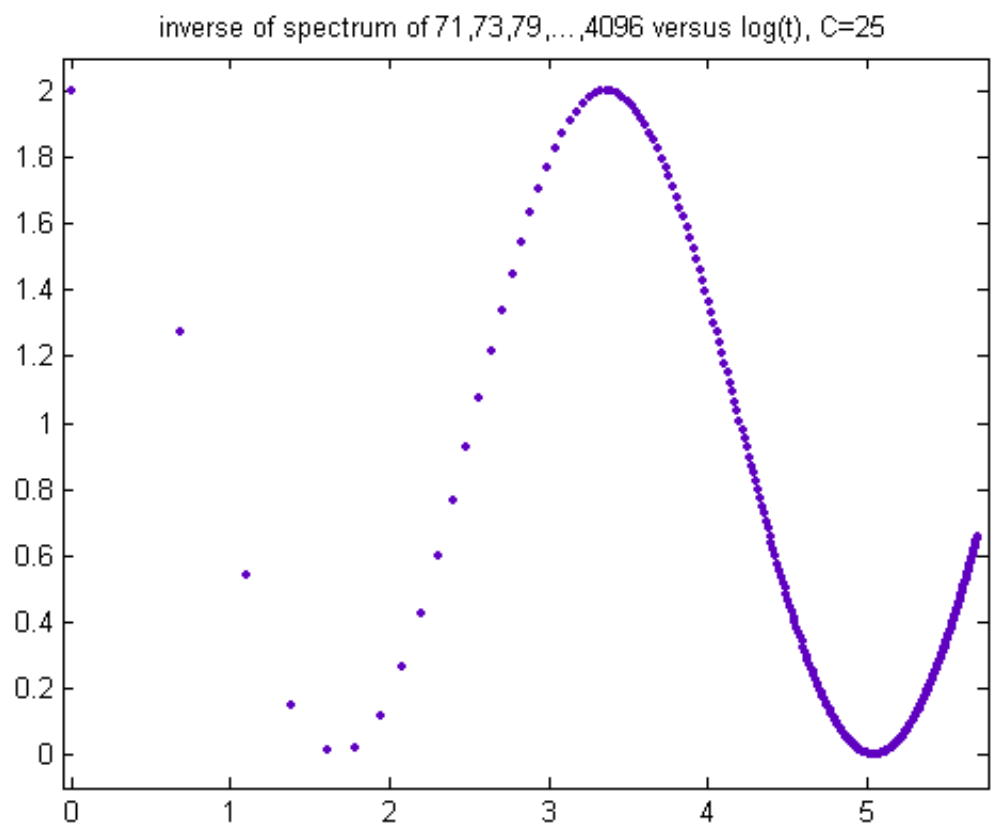

Figure 17: inverse of spectrum

A plot of the inverse function for $r_{n, 13}$ and $t \leq 300$ is 


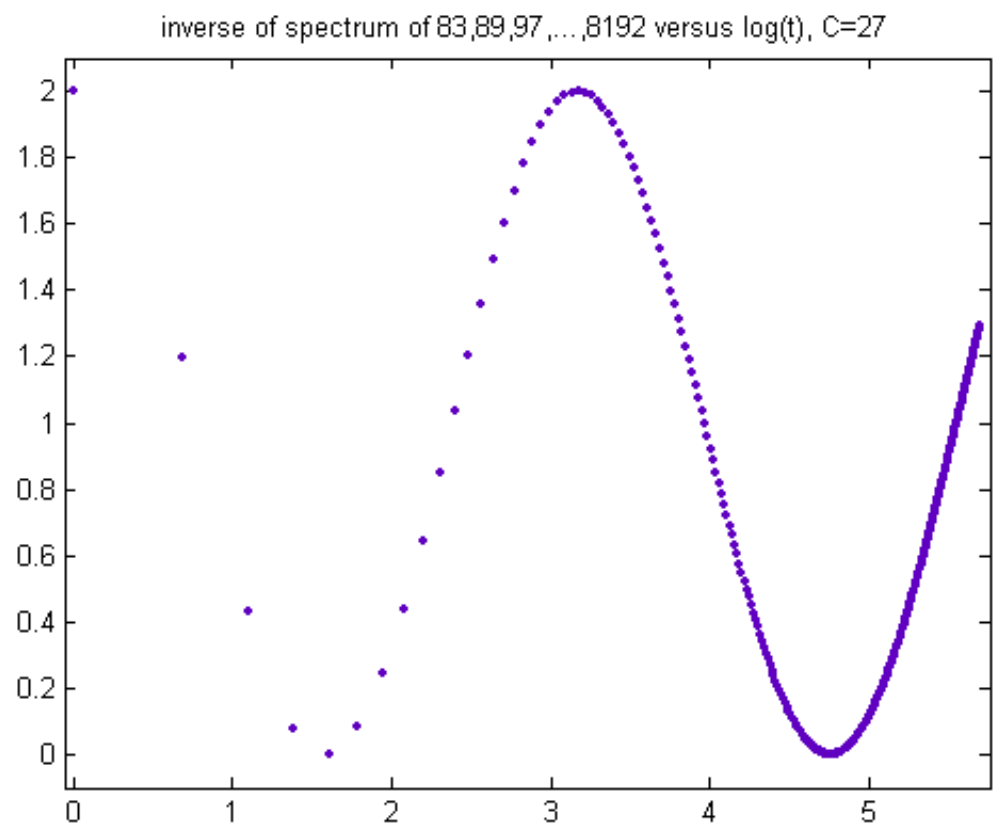

Figure 18: inverse of spectrum

A plot of the inverse function for $r_{n, 14}$ and $t \leq 300$ is 


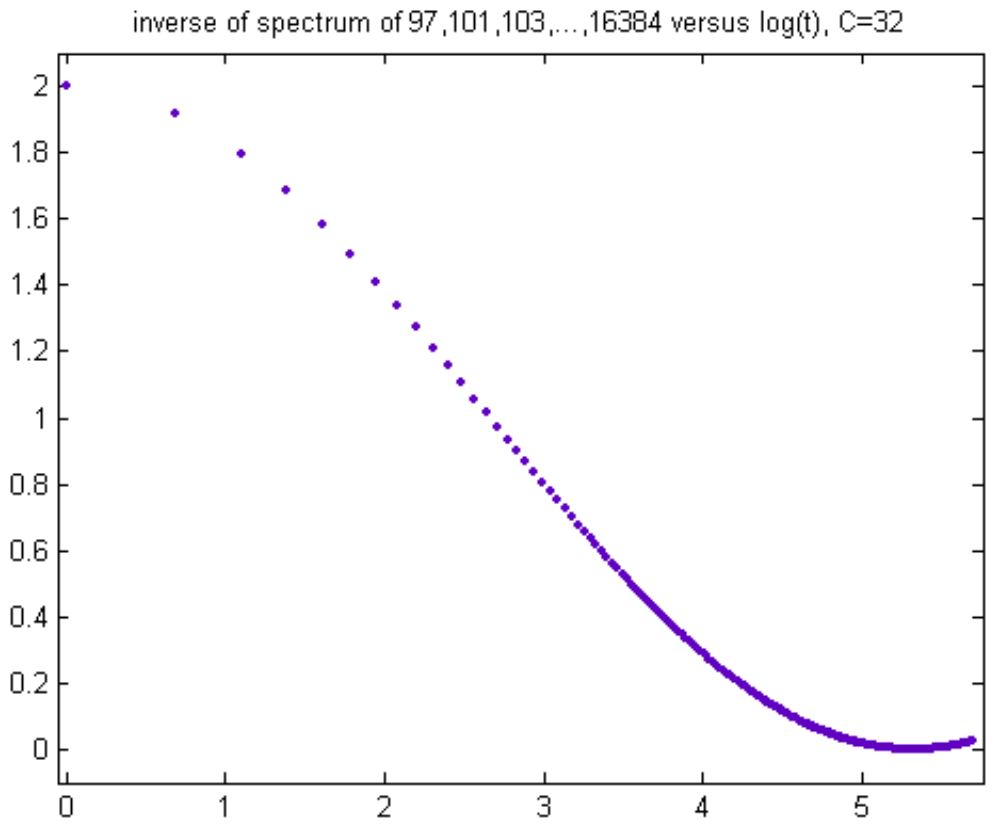

Figure 19: inverse of spectrum

A plot of the inverse function for $r_{n, 15}$ and $t \leq 300$ is 


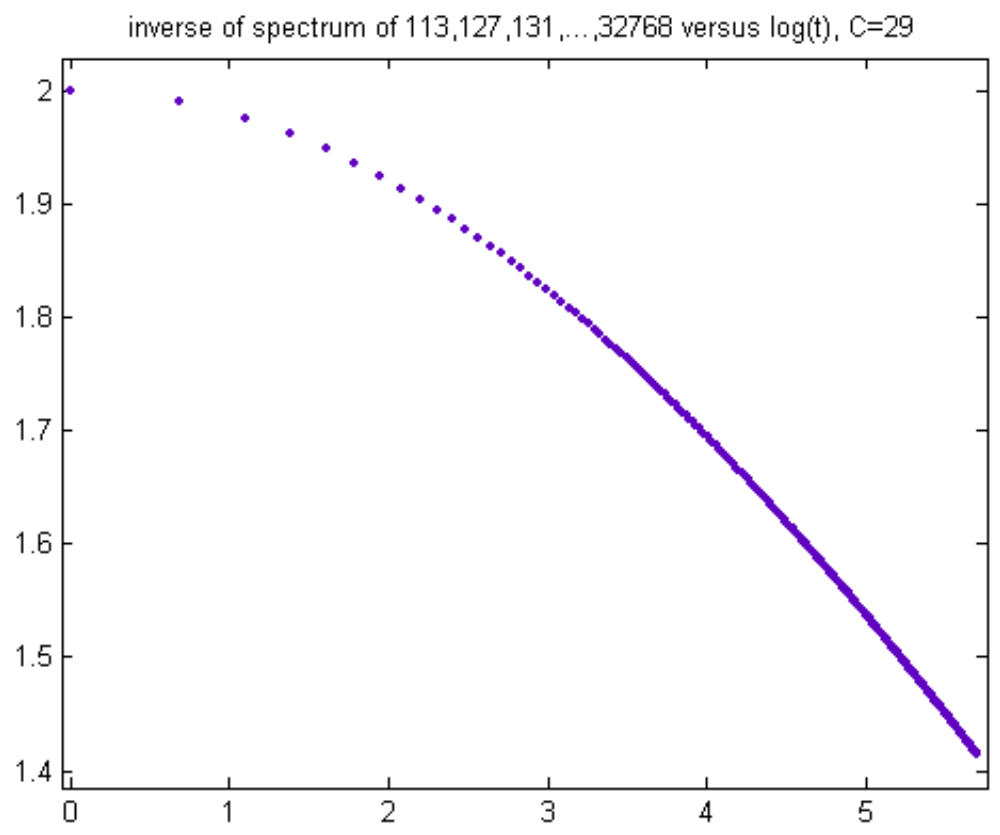

Figure 20: inverse of spectrum

Due to the shape of the curve, the values do not oscillate for larger $t$. If the $C$ value is decremented by 2 , the curve does oscillate but with a large period. A plot of the inverse function for $r_{n, 16}$ and $t \leq 300$ is 


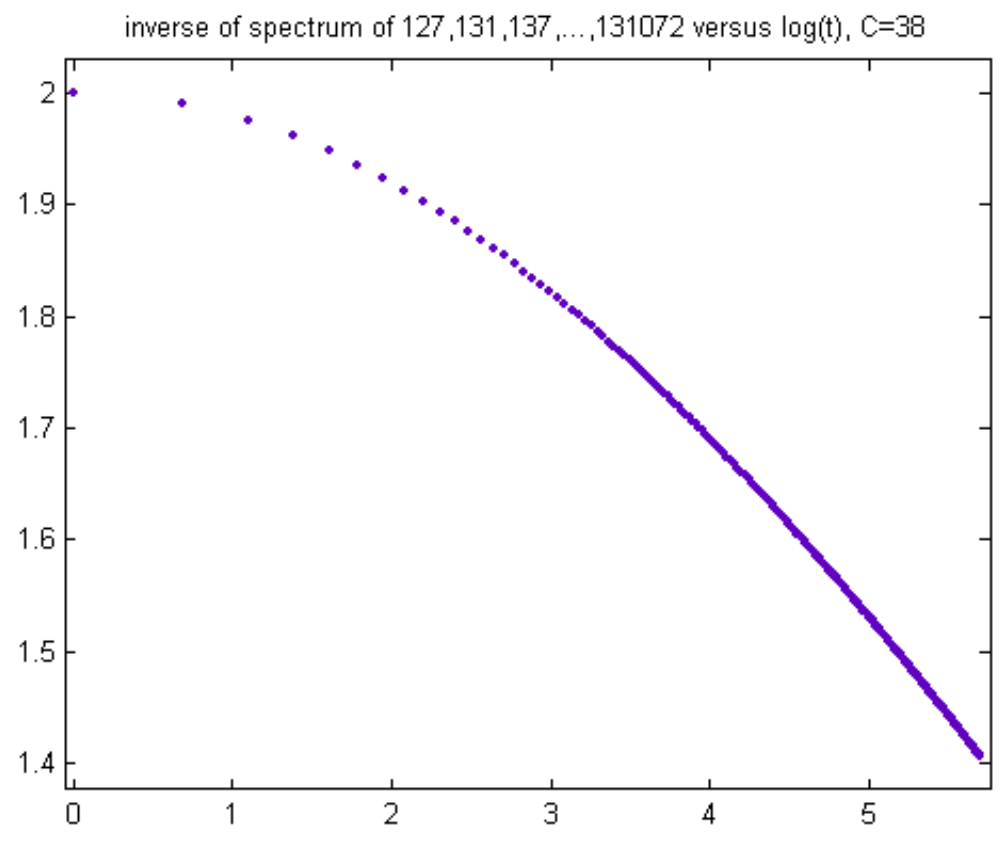

Figure 21: inverse of spectrum

Due to the shape of the curve, the values do not oscillate for larger $t$. If the $C$ value is decremented by 1 , the curve does oscillate but with a fairly large period. A plot of the inverse function of $2,3,4,5,7,8,9,11,13,17,19,23,25$, $27,29,31,32,37,41,43,47,49,53,59,61,64,67,71$, and 73 for $t \leq 300$ is 


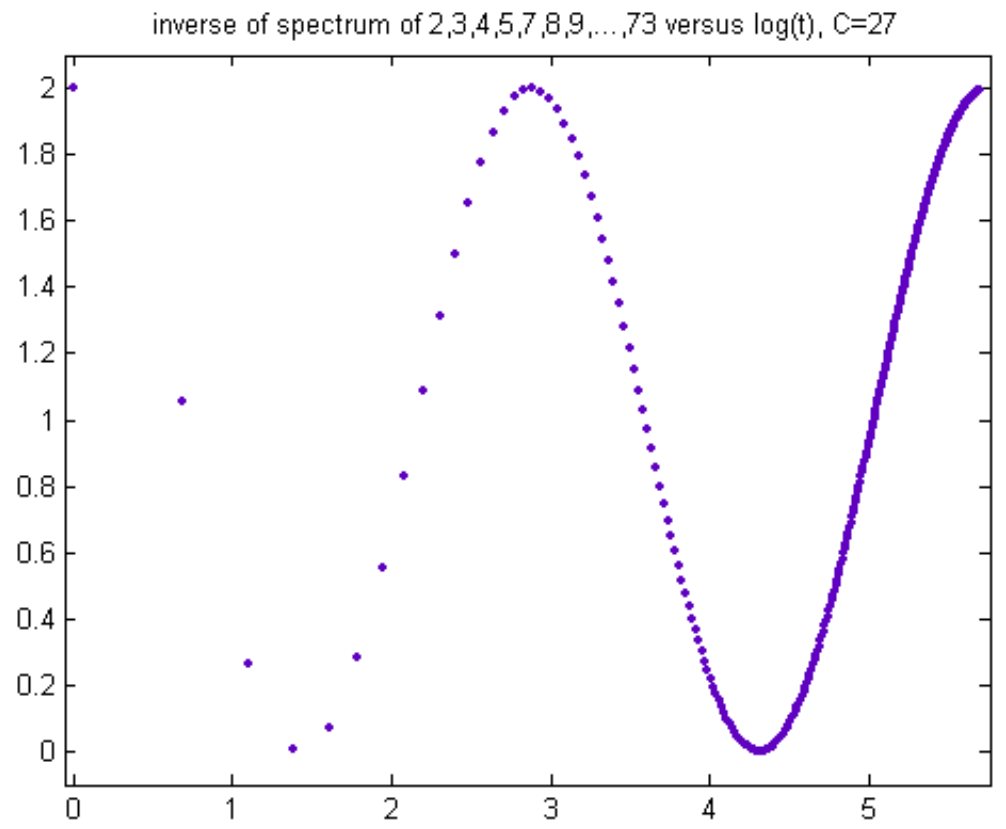

Figure 22: inverse of spectrum

At least the method is useful for comparing spectra. The spectrum of the usual sequence of prime powers compares to the spectrum of $r_{n, 13}$ fairly well. It's not likely that the number of odd numbers in these groups will stop increasing, so perhaps there is another variant of the Riemann hypothesis in the offing. Washington and Yang [5] investigated odd colossally abundant numbers.

Colossally abundant numbers are those numbers $n$ for which there is a positive exponent $\epsilon$ such that $\frac{\sigma(n)}{n^{1+\epsilon}} \geq \frac{\sigma(k)}{k^{1+\epsilon}}$ for all $k>1$. Colossally abundant numbers consist of a product of all the small primes up to some bound, with exponents which are non-increasing as the primes increase. The first few colossally abundant numbers are 2, 6, 12, 60, 120, 360, 2520, 5040, 55440, 720720, 1441440, $4324320,21621600,367567200, \ldots$.

\section{Relevance to the Riemann Hypothesis}

Robin's [6] theorem states that the Riemann hypothesis is true if and only if $\sigma(n)<e^{\lambda} n \log \log n$ for all $n \geq 5041$. Robin showed that if the Riemann hypothesis is false, there will necessarily exist a counterexample to this inequality which is a colossally abundant number. 


\section{References}

[1] T. M. Apostol, Introduction to Analytic Number Theory, Springer, (1976)

[2] G. H. Hardy and E. M. Wright, An Introduction to the Theory of Numbers, Oxford at the Clarendon Press, (1960)

[3] L. Alaoglu and P. Erdös, On Highly Composite and Similar Numbers, Transactions of the American Mathematical Society, Vol. 56, No 3, pp. 448-469, November, 1944

[4] B. Mazur and W. Stein, Prime Numbers and the Riemann Hypothesis, Cambridge University Press, (2016)

[5] L. C. Washington and A. Yang, Analogues of the Robin-Lagarias Criterion of the Riemann Hypothesis, Arxiv:2008.04787v1 [math.NT] 11 Aug 2020

[6] G. Robin, Grandes valeurs de la fonction somme des diviseurs et hypothèse de Riemann, J. Math. Pures Appl. 63 (1984), 187-213. 